\title{
Exploración sonográfica del aneurisma aórtico abdominal: lo clásico y lo novedoso
}

Aguilar, Elibeth*

Restrepo, Patricia**

Sánchez, Ennio***

\section{Correspondencia}

Patricia Restrepo

patrykean@gmail.com

* Latam ultrasound clinical education department manager

**Clínica Las Américas AUNA, Medellín, Colombia

***Hospital Universitario de Caracas, Venezuela

Recibido: 26/02/2021

Aceptado: 28/02/2021

En línea: 30/04/2021

Citar como: Aguilar E, Restrepo P, Sanchez E, Exploración sonográfica del aneurisma aórtico abdominal: Io clásico y lo novedoso. Rev Ecocar Pract (RETIC). 2021 (Abril); 4 (1): 53-54. doi: 10.37615/retic.v4n1a15.

Cite this as: Aguilar E, Restrepo P, Sanchez E, Sonographic exploration of the abdominal aortica aneurysm: the classic and the news. Rev Ecocar Pract (RETIC). 2021 (Abril); 4 (1): 53-54. doi: 10.37615/retic.v4n1a15.

\section{Palabras clave \\ $\triangleright$ Aneurisma aórtico abdominal \\ $\triangleright$ Ultrasonido 3D \\ $\triangleright$ Aorta abdominal

Keywords

$\triangleright$ Abdominal aortic anerysm

$\triangleright$ BD Ultrasound

$\triangleright$ Abdominal aorta

\section{RESUMEN}

Se describen consejos para la evaluación del aneurisma de aorta abdominal y una demostración de la utilidad de la inteligencia artifical

\section{ABSTRACT}

Tips for the evaluation of abdominal aortic aneurysm and a demonstration of the usefulness of artificial intelligence are described.
El aneurisma aórtico abdominal (AAA) es responsable de aproximadamente 175.000 muertes al año de acuerdo con las estadísticas mundiales. Debido a ello, toda evaluación ecocardiográfica debe incluir la valoración de la aorta abdominal vía xifoidea, para descartar esta condición.

\section{Exploración bidimensional del aneurisma aórtico abdominal}

Debe realizarse con el paciente en posición supina, con el transductor en posición transversa a nivel del epigastrio (vista subcostal) y el marcador de la sonda hacia el lado derecho del paciente, para visualizar la aorta proximal y sus estructuras adyacentes; el hígado debe ser la ventana acústica.

Posteriormente, se identifica el cuerpo vertebral, anterior a éste las dos estructuras vasculares anecoicas: a la derecha la vena cava inferior; anterior y hacia la izquierda la aorta. Se examina la aorta proximal, media y distal, transversal y longitudinalmente, hasta la bifurcación de la aorta en las ilíacas.

Se deben obtener imágenes de la aorta tanto en el plano transverso como en el longitudinal, rotando la sonda $90^{\circ}$ en el sentido de las agujas del reloj con el marcador de la sonda hacia la cabeza del paciente (Figuras 1 y 2).

El diámetro de la aorta se debe evaluar en todos sus planos, la manera correcta será midiendo el diámetro desde la pared externa anterior hasta la pared ex- terna posterior, recordando que todas las medidas deben ser perpendiculares al eje de la sonda.

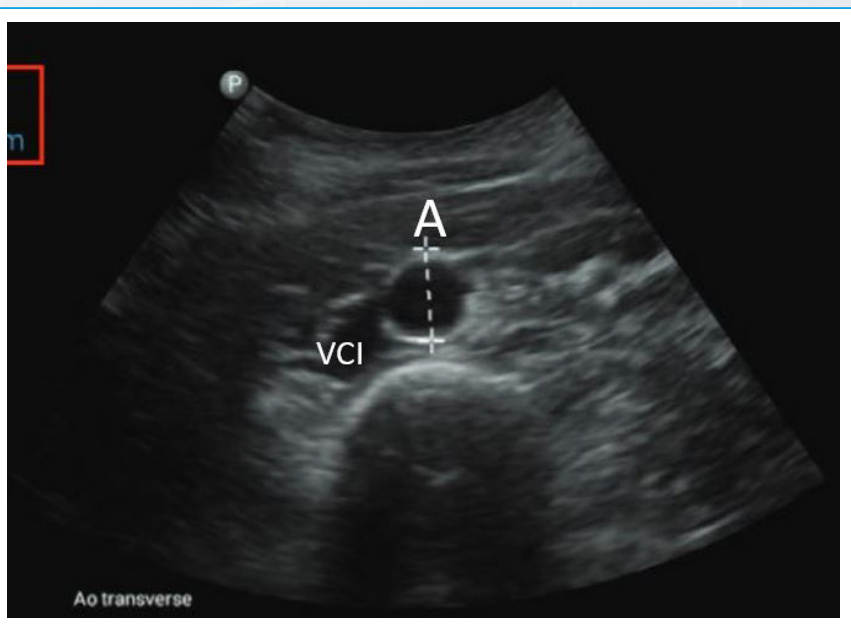

Figura 1. Aorta abdominal, exploración y medición del diámetro transverso

El diámetro normal de la aorta abdominal depende de la edad y el sexo del individuo (es menor en mujeres y jóvenes), siendo el diámetro promedio para adultos alrededor de $2 \mathrm{~cm}$. Un aumento en diámetro mayor de 1,5 veces del valor normal, es decir, un diámetro mayor de $3 \mathrm{~cm}$, se considera patológico. 


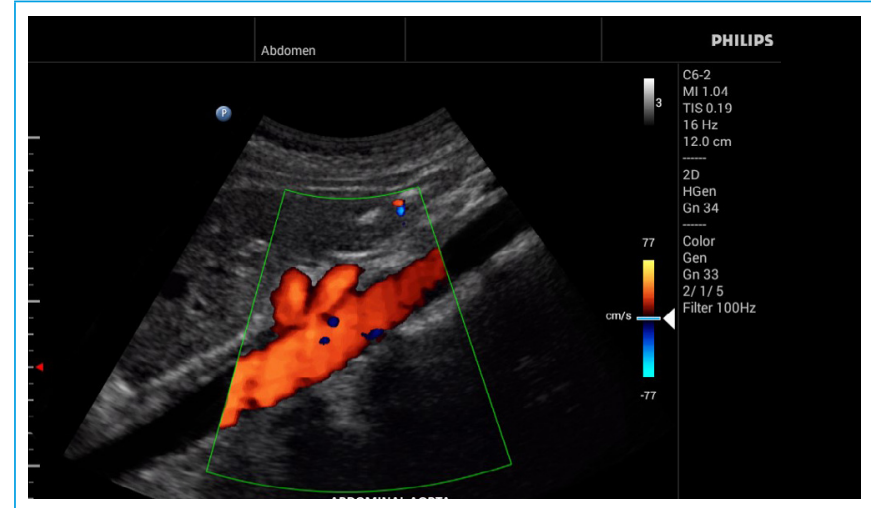

Figura 2. Aorta abdominal, exploración y medición del diámetro longitudinal. Se observa la emergencia de troncos celíacos

\section{Exploración con inteligencia artificial del aneurisma aórtico abdominal}

En esta revisión se trabajó con una aplicación basada en inteligencia artificial (IA), Ilamada "AAA Model", que utiliza un algoritmo el cual presenta automáticamente el análisis del diámetro y volumen del AAA en diferentes planos, permitiendo hacer la edición de los bordes manualmente y reduciendo la variabilidad interoperador.

Para la adquisición, después de optimizar la imagen, se debe comenzar en el plano transverso (Figura 3), asegurando que al activar el software la imagen del aneurisma en ambos planos esté ubicada en el centro y que sea visible la pared posterior de la aorta. Posteriormente, se adquiere el volumen y se verifican los bordes.

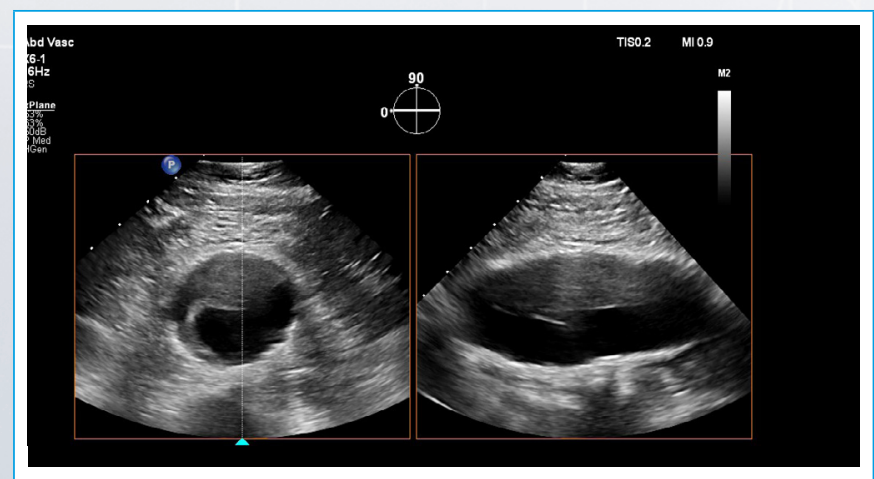

Figura 3. Aneurisma de aorta abdominal parcialmente trombosado, explorado con X-plane, objetivándose en vistas longitudinal y transversal

El software mostrará tres planos bidimensionales (sagital, transversal y axial) y el volumen (Figura 4).

Se recomienda activar el modelo anatómico, el cual permitirá que el sonografista siempre pueda reconocer su orientación respecto a la imagen nativa. Dicho modelo permite saber que el punto verde representa los pies y el punto amarillo representará la cabeza del paciente (Figura 5).

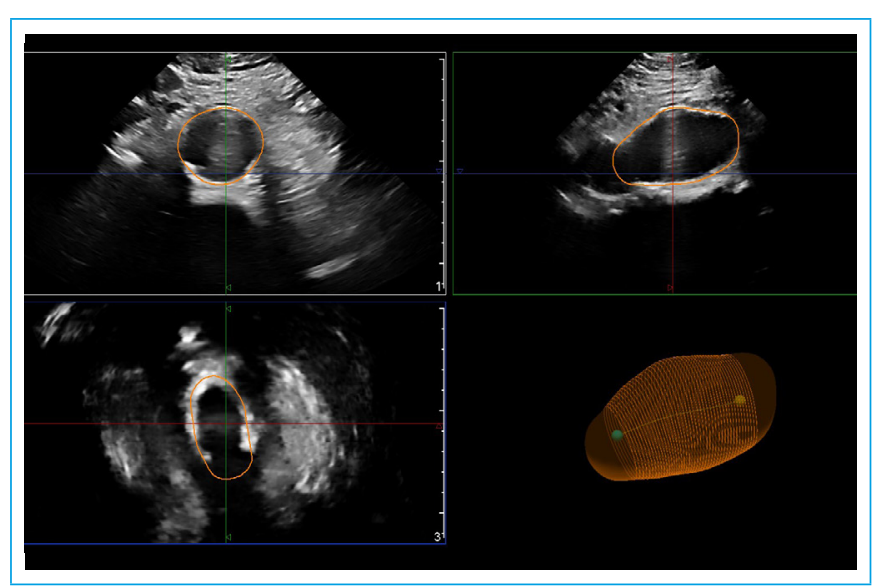

Figura 4. Aorta abdominal, con software de inteligencia artificial que muestra planos bidimensionales (sagital, transversal y axial) y el volumen

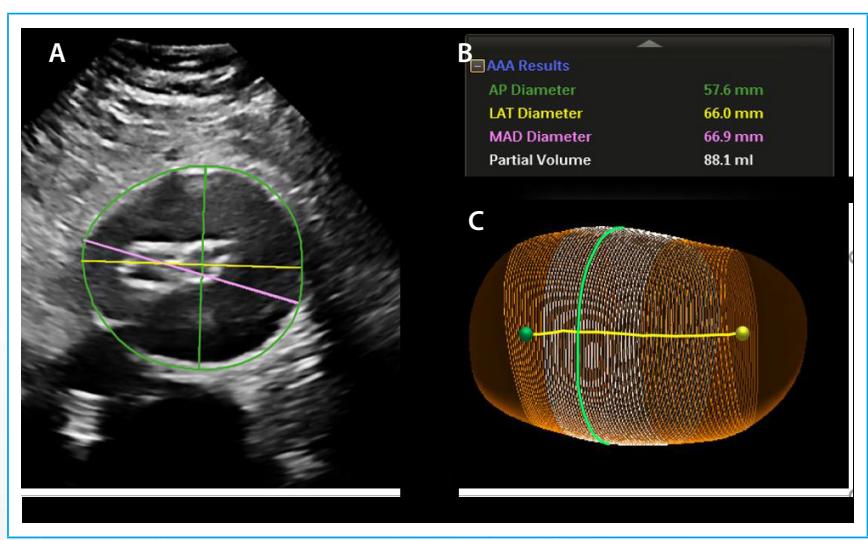

Figura 5. Aorta abdominal, con software de inteligencia artificial.

\section{Ideas para recordar}

- La exploración de la aorta abdominal y detección de aneurisma aórtico mediante ecocardiografía, es un método fácil, rápido y valioso.

- Los avances en inteligencia artificial podrían representar un cambio en la evaluación del aneurisma aórtico abdominal por ultrasonido.

- El uso de la inteligencia artificial da mejores resultados interoperador en la evaluación del aneurisma aórtico abdominal.

\section{Bibliografía}

1. Ghulam QM, Kilaru S, Ou SS and Sillesen H: Clinical validation of three-dimensional ultrasound for abdominal aortic aneurysm. J Vasc Surg. 2019; 71 (1):180-188.

2. Bredahl K, Sandholt B, Lönn L, et al: Three-Dimensional Ultrasound Evaluation of Small Asymptomatic Abdominal Aortic Aneurysms. Eur J Vasc Endovasc Surg. 2015; 49 (3):289-296

3. Ghulam QM, Bredahl KK, Lönn L, et al: Follow-up of Small Abdominal Aortic Aneurysms Using Three-dimensional Ultrasound: Volume Versus Diameter. Eur J Vasc Endovasc Surg. 2017; 54 (4):439-445. 